\title{
PAIN SENSIBILITY IN DEEP SOMATIC STRUCTURES
}

\author{
BY \\ W. H. FEINDEL,* G. WEDDELL, and D. C. SINCLAIR \\ From the Department of Anatomy, University of Oxford
}

(RECEIVED FEBRUARY 20, 1948)

\section{Introduction}

It is well recognized that pain may be provoked by the stimulation of many deep-lying somatic structures, such as muscle, tendon, periosteum, etc. (Lewis, 1942). It was noted by Lewis (1938) that the quality of this pain is independent of the method of stimulation adopted, and that it differs markedly from the quality of the pain which can be aroused from skin. In consequence he suggested that the pain nerves of the skin form a system different from that to which those nerves supplying deeper tissues belong. This contention he supported by observing that the reflexes resulting from painful stimulation of the skin and those due to stimulation of deeplying tissues tended to be of a different character. In 1939, Lewis and Kellgren brought forward additional evidence for the specificity of reflex responses obtained from these two sources. So far, however, no anatomical evidence has been produced which would lend colour to the theory of two separate systems subserving the sensation of somatic pain.

It has been shown (Weddell, 1945) that in normal skin the network of nerve endings subserving the sensation of pain is disposed in such a manner that any one area of skin is supplied by several overlapping terminals derived from different axons. Weddell, Sinclair, and Feindel (1948) have recently investigated the innervation pattern of skin from which an abnormal reaction to painful stimuli was obtained. They found that in such areas the terminals associated with painful sensation were invariably isolated from each other, and that the interweaving of adjacent terminal fibres which is characteristic of the normal skin did not occur. As a result of this investigation they suggested that alterations in the peripheral pattern of innervation of the skin may be responsible for alterations in the quality of the sensation perceived following the application of a painful stimulus. Thus they

* Rhodes Scholar, Nova Scotia, and Merton College, Oxford.

D explained the occurrence of "protopathic" pain in many clinical conditions affecting the peripheral sensory nerves.

From this work it appeared that the difference in quality observed by Lewis (1938) between "skin pain" and "deep somatic pain " might be due to differences between the pattern of innervation in skin and in deeper tissues. Accordingly, it was decided to make an investigation of the innervation patterns in representative deep-lying tissues, and to compare them with the innervation pattern of the skin.

\section{Observations}

Rabbits were perfused with methylene blue solution according to the intravenous drip technique described by Feindel, Sinclair, and Weddell (1947), which allows of the examination of neural patterns in whole tissues. By this means a study was made of the main features of the innervation of skeletal muscle, deep fascia, and periosteum from different regions of the body.

It was found that there is considerable variation between different pieces of the same tissue, more especially when the pieces are derived from different parts of the body. Nevertheless, the main features of the innervation pattern in the three tissues are clearly distinguishable.

(a) Muscle.-In addition to the complex network of motor innervation and the complement of nerve fibres supplying the muscle spindles, all the muscles examined contain a number of fine beaded fibres, which run either in connective tissue septa within the muscle, or as "accessory" fibres to muscle spindles. The general pattern formed by these fine beaded terminals has two main characteristics. The first of these is that these terminals form a very loose meshwork, with relatively large gaps where no fibres or endings are to be found, and the second is that the individual neural terminals in this meshwork are invariably isolated from each other (Figs. 
1 and 2). There is thus no interweaving between adjacent terminal fibres such as occurs in the case of similar fibres in the skin (Fig. 7), nor does superimposition of the territory of one fibre upon that of another occur.

(b) Fascia.-In the portions of deep fascia studied, a similar but rather more dense network of fine beaded fibres occurs; this network is, however, much less closely woven than that characteristic of the innervation of the skin. Though individual fibres are generally isolated from their neighbours (Fig. 3), in a few cases a certain amount of interweaving takes place (Fig. 4), and the territories of such interweaving fibres may overlap each other.

(c) Periosteum.- In the portions studied, the meshwork of beaded fibres tends to be more dense than in either muscle or fascia. Interweaving and superimposition of adjacent terminals is relatively common in these preparations (Figs. 5 and 6), but is still infrequent when compared with conditions in the skin.

\section{Discussion}

In each of the three tissues studied a network of fine beaded fibres has been demonstrated, the density and complexity of which are more evident in periosteum than in muscle, fascia being intermediate in this respect. Such networks have been observed by Hinsey (1927), Denny-Brown (1928) and Weddell and others (1940) in skeletal muscle, and by Weddell and Harpman (1940) in the case of deep fascia, tendon, and periosteum. The fibres composing the networks are exactly similar to those which have been shown to mediate nociceptive responses in the skin of the rabbit's ear (Woollard, Weddell, and Harpman, 1940). These observers showed that terminals of this type persisted in the skin after sympathectomy, and that they occurred in situations remote from blood vessels. Weddell (1941) reported that in a patient with a sciatic nerve injury an area of skin from which pain only could be elicited was examined histologically and found to contain only fine beaded fibres of this type. It is also perhaps worth remarking that the main, if not the only, sensation which can be elicited from the centre of the cornea is one of pain, and that the innervation of this region is largely composed of fine beaded fibres (Feindel, Sinclair, and Weddell, 1947). It is therefore legitimate to assume that neural networks similar to those described in the present paper are résponsible for both nociceptive responses in animals and sensations of pain in man.

It cannot, however, be regarded as established that all the fibres composing these networks are capable of conveying nociceptive impulses, and many of those which run close to blood vessels may in reality be vasomotor fibres. Nevertheless, mere O proximity to a blood vessel does not necessarily $Z$ imply vasomotor function, since Hinsey (1927) and $\stackrel{\Phi}{=}$ Denny-Brown (1928) showed that after sympathectomy a number of fine non-medullated fibres persist $\subseteq$ in relation to the blood vessels in skeletal muscle. Woollard, Weddell, and Harpman (1940) obtained on similar results in the skin of the rabbit's ear, and $\frac{O}{5}$ were able to show that these remaining fibres are capable of mediating nociceptive responses.

Inman and Saunders (1944) injected irritant solutions into various deep somatic structures in $\stackrel{\vec{F}}{\stackrel{+}{9}}$ volunteers, and give a list of tissues in the order of $\bar{C}$ their sensitivity to this procedure. They note that periosteum is the most sensitive, followed by $\frac{\bar{\sigma}}{\vec{D}}$ ligaments; fibrous joint capsules, tendons, fascia, $\stackrel{\square}{\varrho}$ and skeletal muscle. These observations could $\%$ readily be explained on the basis of differences in $\vec{O}$ the density of innervation of the tissues concerned. It should be noted, however, that while the histological evidence obtained in the present investigation is in conformity with the generalization of Inman and Saunders, sufficient variation was observed among the different tissues studied to render it likely that a broad statement of this kind might occasions ally have to be qualified in order to cover compari $\vec{\omega}$ sons between individual regions of the body. It is however, well known to everyone who has given $\frac{0}{8}=$ number of intramuscular injections that quite large $\frac{3}{3}$ needles may often be inserted into muscle withoug causing any pain (Lewis, 1942), whereas periosteurn is notoriously sensitive to such stimulation.* the other hand, a painful response is readily pro $=$ voked if an irritant is injected into muscle. Kellgren (1938) found that $0.3 \mathrm{c.cm}$. of methylene blue solution injected into muscle stains a volume of some $6 \mathrm{c.cm}$. of tissue, and it is therefore clear that the injection of irritant substances provides a diffuse stimulus which is more efficacious than a punctate stimulus in affecting a loose network of nerve terminals such as occurs in muscle.

Kellgren (1938) notes that pain aroused from fascia and from tendon sheaths is moderately well localized, whereas that from muscle is not. This observation also can be explained by differences in the density of innervation (Weddell, 1945), as can the relatively accurate localization of pain aroused from periosteum (Lewis, 1938 ; Kellgren, 1939 ; Weddell and Harpman, 1940). It is maintained by Kellgren (1939) that the capacity to localize pain does not depend primarily on the nature of the tissue stimulated, but is a function of its depth from the surface of the body. The experiments on which

* H. G. Wells (1896) describes a rather more drastic experiment to demonstrate the relative freedom of muscle from nerves capable of transmitting pain. 
this view is founded were carried out with little regard for the local anatomy of the part stimulated, and it seems much more likely that the explanation lies in the differences in the density of innervation of the tissues concerned.

It has been noted that the intimate innervation of the tissues studied differs not only in density but also in pattern from that of the skin. In muscle, the individual nerve terminals are isolated from each other-an arrangement which is only met with in the skin under pathological conditions such as occur in certain scars, and in the territory of regenerating nerves. A painful stimulus applied to such an area of skin may give rise to pain of unpleasant quality, which is imperfectly localized and is often associated with reflex phenomena such as uncontrollable withdrawal, sweating, and dilatation of the pupil, which the same stimulus does not produce when applied to normal skin. Weddell, Sinclair, and Feindel (1948) have attributed this reaction to a reduction in the pattern of impulses reaching the central nervous system . as a result of the isolation of the nerve terminals, and this hypothesis has been shown to provide an adequate explanation in many types of clinical disorder associated with an unpleasant affective reaction to painful stimuli. It may also be noted here that pain which has many of the characteristics of deep somatic pain can be elicited from the skin of a limb in which the circulation has been arrested for some time by means of a compression cuff (Weddell, Sinclair, and Feindel).

There is thus no difficulty in supposing that the " disagreeable" quality characteristic of pain aroused from deep somatic structures (Lewis, 1938) might be due merely to the observed differences between the pattern of innervation in the deep structures and in the skin.

Lewis (1938) made a distinction between the quality of pain aroused from muscle and that resulting from the stimulation of tendon, periosteum, or the web of a finger, but, owing to the difficulty with which this distinction could be recognized, it was subsequently discarded (Lewis and Kellgren, 1939). Weddell and Harpman (1940) noted that they were able to arouse pain of two main varieties from deep fascia and periosteum, one of these being similar to the "first pain" (Lewis, 1942) resulting from a pin-prick on the skin, and the other being unpleasant, diffuse, and of slower development. It is interesting to correlate these observations with the finding that in these tissues a proportion of the network of beaded fibres has the same general features as the network occurring in normal skin, and might therefore be responsible for the occurrence of "first pain." The remainder of the network, consisting of isolated terminals, might give rise to pain of the " disagreeable" quality described by Lewis (1938). It is curious, however, that Weddell and Harpman (1940) found that from tendon substance only one type of pain could be elicitedthe sharp pricking pain corresponding to "first pain." This circumstance is perhaps to be explained by the fact that the observations were made on the tendo Achillis, which has a peculiarly intimate relationship to the cutaneous nerve fibres supplying the skin overlying it ; it is possible that these fibres were not adequately anæsthetized. Lewis (1942), on the other hand, notes that pain aroused by irritant injections into tendon substance is of the characteristic quality associated with deep somatic pain.

Lastly, it may be noted that the distinctions which Lewis (1938) and Lewis and Kellgren (1939) make between the reflexes aroused from deep and superficial somatic structures by painful stimulation do not appear to be of a fundamental nature. Such differences as occur may be easily explained, since the pattern of impulses reaching the central nervous system must be different in the two cases, and it is not only possible, but likely, that such different patterns should be capable of giving rise to different reflex responses.

In short, it appears that the observed differences between pain produced from the skin and that due to the stimulation of deep somatic structures can be satisfactorily explained without the necessity of postulating the existence of two separate painconducting systems of nerves.

\section{Summary and Conclusions}

The hypothesis of Lewis (1938) that the skin and the deeper somatic tissues are supplied by two separate systems of pain nerves has been examined in the light of the recent work of Weddell, Sinclair, and Feindel (1948), who found that alterations in the quality of pain sensibility can be produced by alterations in the pattern of the pain-producing impulses reaching the central nervous system. A preliminary examination of the innervation of certain deep somatic structures has been made, and it has been found that this innervation differs both in pattern and in density from that of the skin. These findings are sufficient to explain the observed differences in the subjective and objective results of painful stimulation of the skin and of deeper lying structures respectively. There is thus no need to postulate two separate pain-conducting systems supplying somatic tissues.

The expenses of this investigation were defrayed by a grant to one of us (G.W.) from the Medical Research Council, which is most gratefully acknowledged. 


\section{REFERENCES}

Denny-Brown, D. (1928). " "On the Essential Mechanism of Mammalian Posture." Oxford University D.Phil. Thesis.

Feindel, W. H., Sinclair, D. C., and Weddell, G. (1947). Brain, 70, 495 .

Hinsey, J. C. (1927). J. comp. Neurol., 44, 87.

Inman, V. T., and Saunders, J. B. de C. M. (1944). J. nerv. ment. Dis., 99, 660.

Kellgren, J. H. (1938). Clin. Sci., 3, 175. (1939). Ibid., 4, 35.

Lewis, T. (1938). Brit. med. J., 1, 321. (1942). "Pain." New York. Macmillan.
Lewis, T., and Kellgren, J. H. (1939). Clin. Sci., 4, 47.

Weddell, G. (1941). . J. Anat., Lond., 75, 346. (1945). Brit. med. Bull., 3, 167.

-, and Harpman, J. A. (1940). J. Neurol. Psychiat., 3, 319.

$\longrightarrow$, Sinclair, D. C., and Feindel, W. H. (1948). J. Neurophysiol. (In the Press.)

- Harpman, J. A., Lambley, D. G., and Young, L. (1940). J. Anat., Lond., 74, 255.

Wells, H. G. (1896). "The Island of Dr. Moreau." London. Heinemann.

Woollard, H. H., Weddell, G., and Harpman, J. A. (1940). J. Anat., Lond., 74, 413.

\section{Legends to Illustration}

FIGS. 1 to 6 are from methylene blue preparations in the rabbit.

FIG. 1.-Fine beaded nerve fibre related to a muscle spindle in a lumbrical muscle. Note the absence of interweaving and the isolation of the fibre (cf. Fig. 7). A motor end-plate is seen in relation to the darkly stained intrafusal muscle fibre. $(\times 1000$.

FIG. 2.-Fine beaded nerve fibre in a lumbrical muscle. The fibres in both Fig. 1 and Fig. 2 have no relation to blood-vessels. ( $\times$ 1000.)

FIG. 3.-Isolated beaded fibre in fascial sheath of neck muscle. Compare with Figs. 4 and 7. ( $\times$ 850.)

FIG. 4.-Interweaving nerve fibres from another part of the same specimen as shown in Fig. 3. The arrangement is less complex than that shown in Fig. 7. ( $\times$ 1100.)

FIGS. 5 and 6.-Show respectively an isolated beaded fibre from the periosteum of the tibia, and a pair of interweaving fibres from another part of the same specimen. ( $\times$ 350.)

Fig. 7.-Leash of fine beaded nerve fibres in human skin from the chest removed following local injection of methylene blue. ( $\times$ 950.) Note interweaving of adjacent fibres.

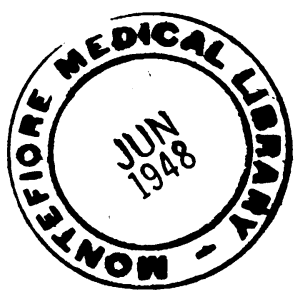




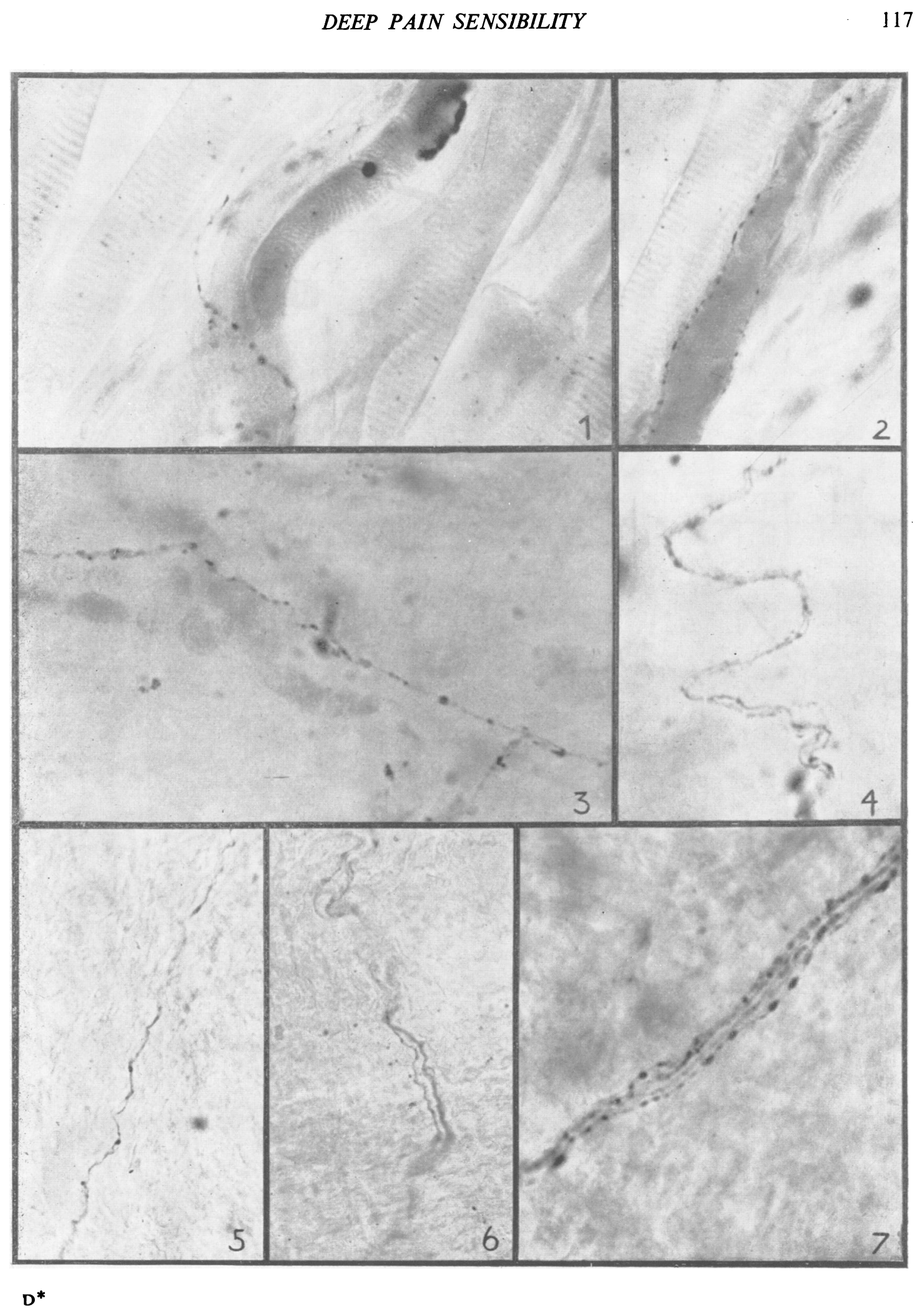

